\title{
ORDINARY SUBVARIETIES OF CODIMENSION ONE
}

\author{
FERRUCCIO ORECCHIA
}

\begin{abstract}
In this paper we extend the properties of ordinary points of curves [10] to ordinary closed points of one-dimensional affine reduced schemes and then to ordinary subvarieties of codimension one.
\end{abstract}

\section{INTRODUCTION.}

Let $A$ be the local ring, at a point $x$, of an algebraic reduced variety $X$ over an algebraically closed field $k$. Denote with $e(A)=e$ the multiplicity of $X$ at $x$. Let $\mathfrak{m}$ be the maximal ideal of $A$. $\operatorname{Spec}(G(A))$ is the tangent cone and $\operatorname{Proj}(G(A))$ the projectivized tangent cone to $X$ at $x$. Furthermore, if $G(A)_{\text {red }}=\operatorname{Spec}(G(A) / \operatorname{nil}(G(A))$, then $\operatorname{Spec}\left(G(A)_{\text {red }}\right)$ is the tangent cone to $X$ at $x$, viewed as a set. If $\bar{A}$ is the normalization of $A$, the branches of $X$ at $x$ are the points of $\operatorname{Spec}(\bar{A} / \mathfrak{m} \bar{A})$.

If $A$ is the local ring, at a point $x$, of a curve $C$ and $\mathfrak{p}_{i}, i=1, \ldots, n$, are the minimal primes of $G(A)$ then $\operatorname{Spec}\left(G(A) / \mathfrak{p}_{i}\right)$ are the tangents of $C$ at $x$ (that is the tangents are the lines of $\operatorname{Spec}\left(G(A)_{\text {red }}\right)$ and correspond to the points of $\left.\operatorname{Proj}(G(A))\right)$. It is well known [10, Lemma-Definition 2.1] that each branch has a tangent and that the following conditions are equivalent:

(a) The scheme $\operatorname{Proj}(G(A))$ is reduced;

(b) $\operatorname{Proj}(G(A))$ consists of $e$ points;

(c) there are $e=e(A)$ tangents at $x$;

(d) the curve $C$ has, at $x, e$ linear branches with distinct tangents.

A point $x$ which satisfies these conditions is said to be an ordinary point. Any nonsingular point of the curve $(e=1)$ is a trivial example of ordinary point. Generally, but not always [9, Section 4], an ordinary point has reduced tangent cone, in the sense that, if its tangents are in generic position, then the tangent cone to $C$ at $x$ (i.e. $G(A)$ ) is reduced [10, Theorem 3.3].

In this paper we extend these results to subvarieties of codimension one.

For this we need first to extend the notion of ordinary point of a curve to the notion of ordinary closed point of a one-dimensional reduced local affine scheme $\operatorname{Spec}(A)$. In fact let $\mathfrak{m}$ be the maximal ideal of $A$ and $K$ be the algebraic closure of the residue field $k(\mathfrak{m})$ of $A$. Then the closed point of $\operatorname{Spec}(A)$ is said to be ordinary if $\operatorname{Proj}\left(G(A) \otimes_{k(\mathfrak{m})} K\right)$ is reduced [Definition 2.4].

1991 Mathematics Subject Classification. 13A30.

Key words and phrases. Generic position, ordinary multiple subvarieties, conductor.

Work partially supported by MURST 
We prove that the previous equivalences $(a) \Leftrightarrow(b) \Leftrightarrow(c) \Leftrightarrow(d)$ continue to hold in this more general setting if we suitably define (geometric) tangents and (geometric) branches and we prove that if the ordinary closed point of $\operatorname{Spec}(A)$ has (geometric) tangents in generic position then $G(A) \otimes_{k(\mathfrak{m})} K$ is reduced.

Then, basing on this, using results of [2] and the notion of normal flatness, we extend the properties of ordinary points to subvarieties of codimension one. Let $X=\operatorname{Spec}(R)$ be an equidimensional algebraic variety and $Y=\operatorname{Spec}(R / \mathfrak{q})$ be an irreducible codimension one subvariety of $X$ of multiplicity $e=e\left(R_{\mathfrak{q}}\right)$. If $A$ is the local ring of $X$ at a closed point $x$ of $Y$ then $x$ is said to be an ordinary point if $\operatorname{Spec}\left(G(A)_{\text {red }}\right)$ is the union of $e$ distinct linear spaces. $Y$ is said to be an ordinary subvariety of $X$ if the closed point of the one-dimensional scheme $\operatorname{Spec}\left(R_{\mathfrak{q}}\right)$ is ordinary. Assume that $Y$ is nonsingular at $x$ and $X$ is normally flat along $Y$ at $x$, then $x$ is an ordinary point of $X$ if and only if $X$ has, at $x, e$ linear branches with distinct tangent spaces. If these tangent spaces are in generic position then $G(A)$ is reduced [Theorem 3.4]. Moreover $Y$ is an ordinary subvariety of $X$ if and only if there exists an open non-empty subset $U$ of $Y$ such that every closed point $x$ of $U$ is ordinary [Theorem 3.5].

This paper has been motivated by [12] in which the conductor of a variety with ordinary (multiple) subvarieties of codimension one is computed. We would like to thank G. Castaldo for pointing out the paper [2].

Throughout the paper all rings are supposed to be commutative, with identity and noetherian.

Let $A$ be a local ring with maximal ideal $\mathfrak{m}$. With $H^{0}(A, n)=\operatorname{dim}_{k}\left(\mathfrak{m}^{n} / \mathfrak{m}^{n+1}\right)$, $n \in \mathbb{N}$, we denote the Hilbert function of $A$ and with $e(A)$ the multiplicity of $A$ at $\mathfrak{m}$. The embedding dimension $\operatorname{emdim}(A)$ of $A$ is given by $H^{0}(A, 1)$. With $A^{h}$ we denote the henselization of $A$ and with $A^{\text {hs }}$ a fixed strict henselization of $A$ (see $\left[6\right.$, Number 32, Section 18] or [13]). If $\mathfrak{p}$ is an ideal of $A, G_{\mathfrak{p}}(A)=\bigoplus_{n \geq 0}\left(\mathfrak{p}^{n} / \mathfrak{p}^{n+1}\right)$ is the associated graded ring with respect to $\mathfrak{p}$. By $G(A)$ we denote the associated graded ring with respect to $\mathfrak{m}$. $\operatorname{Spec}(G(A)$ is the tangent cone to $\operatorname{Spec}(A)$ at its closed point. If $S=\bigoplus_{n \geq 0} S_{n}$ is a standard graded finitely generated algebra over a field $k$, with maximal homogeneous ideal $\mathfrak{n}, H(S, n)=\operatorname{dim}_{k} S_{n}=H\left(S_{\mathfrak{n}}, n\right)$ denotes the Hilbert function of $S$ and $\operatorname{emdim}(S)=H(S, 1)=\operatorname{emdim}\left(S_{\mathfrak{n}}\right)$ denotes the embedding dimension of $S$. The multiplicity of $S$ is $e(S)=e\left(S_{\mathfrak{n}}\right)$. On has $e(A)=e(G(A))$ and $\operatorname{emdim}(A)=\operatorname{emdim}(G(A))$. If $k(\mathfrak{m})$ is the residue field of $A$, $\operatorname{Hom}_{k(\mathfrak{m})}\left(\mathfrak{m} / \mathfrak{m}^{2}, k(\mathfrak{m})\right)$ is the tangent space of $\operatorname{Spec}(A)$ at its closed point. If $A$ is regular then the tangent cone and the tangent space of $A$ coincide.

If $R$ is any ring $\operatorname{dim}(R)$ denotes the dimension of $R$.

\section{Generalities on BRAnChes And nORmal flatness}

Definition 1.1. Let $A$ be a local reduced ring with maximal ideal $\mathfrak{m}$. Let $K$ be the algebraic closure of the residue field $k(\mathfrak{m})=A / \mathfrak{m}$ and $\bar{A}$ be the normalization of $A$. A branch of $\operatorname{Spec}(A)$ at its closed point is a point of $\operatorname{Spec}(\bar{A} / \mathfrak{m} \bar{A})$. A geometric branch of $\operatorname{Spec}(A)$ at its closed point is a point of $\operatorname{Spec}\left(\bar{A} / \mathfrak{m} \bar{A} \otimes_{k(\mathfrak{m})} K\right)$. If $X$ is a scheme a branch (respectively a geometric branch) of $X$ at a closed point $x$ is a branch (respectively a geometric branch) at the closed point of $\operatorname{Spec}(A)$, where $A$ 
Remark. Since $\bar{A}$ is integral over $A$ the $\operatorname{ring} \bar{A} / \mathfrak{m} \bar{A}$ is zero-dimensional and then the $\operatorname{ring} \bar{A} / \mathfrak{m} \bar{A} \otimes_{k(\mathfrak{m})} K$ is zero-dimensional [6, $N^{0} 24$, Corollaire 4.1.4]. Then the points of the schemes $\operatorname{Spec}(\bar{A} / \mathfrak{m} \bar{A})$ and $\operatorname{Spec}\left(\bar{A} / \mathfrak{m} \bar{A} \otimes_{k(\mathfrak{m})} K\right)$ are closed.

Lemma 1.2. If $A$ is a local reduced ring, there is a canonical bijection between the set of branches of $\operatorname{Spec}(A)$ and the set of minimal primes of $A^{h}$ and there is a canonical bijection between the set of geometric branches of $A$ and the set of minimal primes of $A^{\text {sh }}$.

Proof. [5, Theorem 2.1]

Definition 1.3. Let $A$ be a local reduced ring and $\gamma$ be a branch (respectively a geometric branch) of $\operatorname{Spec}(A)$ at its closed point. If $\mathfrak{p}$ is the minimal prime of $A^{h}$ (respectively $A^{h s}$ ) corresponding to $\gamma$, the order of the branch (respectively the order of the geometric branch) $e(\gamma)$ is the multiplicity $e\left(A^{h} / \mathfrak{p}\right)$ (respectively $\left.e\left(A^{s h} / \mathfrak{p}\right)\right)$. The branch (respectively the geometric branch) $\gamma$ is linear if $A^{h} / \mathfrak{p}$ (respectively $A^{\text {hs }} / \mathfrak{p}$ ) is a regular ring.

Remark. The order of a geometric branch is independent from the strict henselization of $A$ chosen [1, Corollary 1.9]

Proposition 1.4. Let $A$ be a reduced local ring of dimension one or the local ring at a closed point of an algebraic equidimensional reduced variety (over an algebraically closed field $k$ ). Let $\gamma_{i}, i=1, \ldots, n$ be the branches (respectively the geometric branches) of $\operatorname{Spec}(A)$ at the closed point. Then:

(a) $e(A)=\sum_{i=1}^{n} e\left(\gamma_{i}\right)$;

(b) $\gamma_{i}$ is linear if and only if its order is one;

(c) $n=e(A)$ if and only if all the branches are linear.

Proof. (a), (b) (see [1, Propositions 2.7 and 2.10]). (c) is obvious consequence of (a) and $(b)$.

Definition 1.5. Le $\mathfrak{p}$ be a prime ideal of a local ring $A$. $A$ is normally flat along $\mathfrak{p}$ if $\mathfrak{p}^{n} / \mathfrak{p}^{n+1}$ is flat over $A / \mathfrak{p}$, for all $n \geq 0$. Note that $A$ is normally flat along $\mathfrak{p}$ if and only if $G_{\mathfrak{p}}(A)$ is free over $A / \mathfrak{p}$.

Theorem 1.6. Let $A$ be a local ring and $\mathfrak{p}$ a prime ideal of $A$ such that $A / \mathfrak{p}$ is regular of dimension $d$ and $A$ is normally flat along $\mathfrak{p}$. Then:

(a) there is an isomorphism of graded $k$-algebras

$$
G(A) \cong\left(G_{\mathfrak{p}}(A) \otimes_{A / \mathfrak{p}} k\right)\left[T_{1}, \ldots, T_{d}\right]
$$

(b) the following rings have the same Hilbert functions:

$G_{\mathfrak{p}}(A) \otimes_{A / \mathfrak{p}} k($ over $k)$ and $G\left(A_{\mathfrak{p}}\right)($ over $k(\mathfrak{p}))$

Proof. (a) [7, Corollary (21.11)].

(b) [8, Chapter II, Corollary 2].

Let $X=\operatorname{Spec}(R)$ be a reduced variety over an algebraically closed field $k$ and 
Definition 1.7. If $x$ is any closed point of $Y, A$ is the local ring of $X$ at $x$ and $\mathfrak{p}=\mathfrak{q} A$ is the prime ideal in $A$ defining the subvariety $Y$, then:

( $i) X$ is normally flat along $Y$ at $x$ if $A$ is normally flat along $\mathfrak{p}$;

(ii) $Y$ is nonsingular at $x$ if $A / \mathfrak{p}$ is regular.

Theorem 1.8. There exists an open nonempty subset $U$ of $Y$ such that, for every closed point $x$ of $U, Y$ is nonsingular at $x$ and $X$ is normally flat along $Y$ at $x$.

Proof. It is well known that the nonsingular points of $Y$ form an open nonempty set and that $X$ is normally flat along $Y$ at the points of an open nonempty subset of $Y$ [7, Corollary (24.5)].

\section{ORdinARY ClOSED POINTS OF ONE DIMENSIONAL AFFINE REDUCED SCHEMES.}

In this section $A$ is a reduced local one-dimensional ring of multiplicity $e=e(A)$ and maximal ideal $\mathfrak{m}$. With $K$ we denote the algebraic closure of the residue field $k(\mathfrak{m})$ of $A$ and with $\bar{A}$ we denote the normalization of $A$.

Lemma 2.1. (a) There is a natural immersion $G(A) \subset G(A) \otimes_{k(\mathfrak{m})} K$;

(b) $G(A) \otimes_{k(\mathfrak{m})} K$ is one-dimensional;

(c) the Hilbert functions of $A, G(A)$ and $G(A) \otimes_{k(\mathfrak{m})} K$ are the same, moreover $e(A)=e(G(A))=e\left(G(A) \otimes_{k(\mathfrak{m})} K\right)$ and $\operatorname{emdim}(A)=\operatorname{emdim}(G(A))=$ $\operatorname{emdim}\left(G(A) \otimes_{k(\mathfrak{m})} K\right)$.

Proof. (a) The immersion follows by the flatness of $G(A)$ over $k(\mathfrak{m})$

(b) $\left[6, N^{0} 24\right.$, Corollaire 4.1.4]

(c) $G(A) \otimes K$ is the $K$-vector space obtained extending to $K$ the field of scalars of the $k(\mathfrak{m})$-vector space $G(A)$, then the Hilbert functions of $G(A)$ (that is of $A$ ) and of $G(A) \otimes K$ are the same. This implies the equalities of the multiplicities and of the embedding dimensions.

Since, by Lemma $2.1, G(A) \otimes_{k(\mathfrak{m})} K$ is a graded one-dimensional algebra over the algebraically closed field $K, \operatorname{Spec}\left(\left(G(A) \otimes_{k(\mathfrak{m})} K\right)_{r e d}\right)$ is a union of lines each corresponding to a point of $\operatorname{Proj}\left(\left(G(A) \otimes_{k(\mathfrak{m})} K\right)\right)$.

Definition 2.2. the lines of $\operatorname{Spec}\left(\left(G(A) \otimes_{k(\mathfrak{m})} K\right)_{\text {red }}\right)$ are the geometric tangents of $\operatorname{Spec}(A)$ at its closed point.

Let $n$ be a positive integer and $\mathfrak{m}^{n}: \mathfrak{m}^{n}=\left\{b \in \bar{A} \mid b \mathfrak{m}^{n} \subset \mathfrak{m}^{n}\right\}$. We recall that the subring of $\bar{A}, B=\cup_{n>0}\left(\mathfrak{m}^{n}: \mathfrak{m}^{n}\right)$, is the ring obtained by blowing up the maximal ideal $\mathfrak{m}$ of $A$.

The natural isomorphism of schemes $\operatorname{Spec}(B / \mathfrak{m} B) \cong \operatorname{Proj}(G(A))\left[6, N^{0} 32\right.$, Lemma 19.4.2] induces an isomorphism of schemes

$$
\phi: \operatorname{Spec}\left(B / \mathfrak{m} B \otimes_{k(\mathfrak{m})} K\right) \longrightarrow \operatorname{Proj}\left(G(A) \otimes_{k(\mathfrak{m})} K\right)
$$

By Definition 1.1 a geometric branch $\gamma$ of $\operatorname{Spec}(A)$ is a point of $\operatorname{Spec}\left(\bar{A} / \mathfrak{m} \bar{A} \otimes_{k(\mathfrak{m})} K\right)$. The inclusion $B \subset \bar{A}$ induces a natural homomorphism of zero-dimensional rings 
and then a morphism

$$
\psi: \operatorname{Spec}\left(\bar{A} / \mathfrak{m} \bar{A} \otimes_{k(\mathfrak{m})} K\right) \longrightarrow \operatorname{Spec}\left(B / \mathfrak{m} B \otimes_{k(\mathfrak{m})} K\right)
$$

Hence $\phi(\psi(\gamma))$ is a point of $\operatorname{Proj}\left(G(A) \otimes_{k(\mathfrak{m})} K\right)$.

Definition 2.3. The line of $\operatorname{Spec}\left(\left(G(A) \otimes_{k(\mathfrak{m})} K\right)_{\text {red }}\right)$ corresponding to $\phi(\psi(\gamma))$ is a geometric tangent of $\operatorname{Spec}(A)$ which we call the geometric tangent to $\gamma$.

Definition 2.4. The closed point of the scheme $\operatorname{Spec}(A)$ is said to be ordinary if $\operatorname{Proj}\left(G(A) \otimes_{k(\mathfrak{m})} K\right)$ is reduced.

Theorem 2.5. The following conditions are equivalent:

(a) the closed point of $\operatorname{Spec}(A)$ is ordinary

(b) $\operatorname{Proj}\left(G(A) \otimes_{k(\mathfrak{m})} K\right)$ consists of e points

(c) there are e geometric tangents to $\operatorname{Spec}(A)$

(d) Spec $(A)$ has e linear geometric branches with distinct geometric tangents.

Proof. $(a) \Leftrightarrow(b)$. Set $R=G(A) \otimes_{k(\mathfrak{m})} K$ and let $\mathfrak{q}_{i}, i=1, \ldots, n$, be the minimal primary ideals of $R$ and $\mathfrak{p}_{i}=\sqrt{\mathfrak{q}_{i}}$ be the corresponding minimal primes. We have to prove that $\operatorname{Proj}(R)$ is reduced if and only if $n=e=e(A)$. But $\operatorname{Proj}(R)$ is reduced if and only if $\mathfrak{q}_{i}=\mathfrak{p}_{i}$, for any $i$. By standard facts on multiplicity we have $e(R)=\sum_{i=1}^{n} \lambda\left(\mathfrak{q}_{i}\right) e\left(R / \mathfrak{p}_{i}\right)=\sum_{i=1}^{n} \lambda\left(\mathfrak{q}_{i}\right)$, where $\lambda\left(\mathfrak{q}_{i}\right)$ denotes the lenght of the ideal $\mathfrak{q}_{i}$ (note that $e\left(R / \mathfrak{p}_{i}\right)=1$ because $\operatorname{Spec}\left(R / \mathfrak{p}_{i}\right)$ is a line). Moreover, by Lemma $2.1,(\mathrm{c}), e(A)=e(R)$ and then the claim follows from the fact that $\lambda\left(\mathfrak{q}_{i}\right)=1$ if and only if $\mathfrak{q}_{i}$ is prime.

$(b) \Leftrightarrow(c)$. Follows immediately from Definition 2.2 .

$(c) \Leftrightarrow(d)$. By definition each geometric branch has a geometric tangent and then the claim is clear if we consider Proposition 1.4,(c)

Example 2.6. Let the normalization $\bar{A}$ be finite over $A$. If $k(\mathfrak{m})$ has characteristic zero and $A$ is a seminormal ring the closed point of $\operatorname{Spec}(A)$ is an ordinary point with $e(A)=e m \operatorname{dim}(A)$ tangents. In fact $A$ is seminormal if and only if $\operatorname{emdim}(A)=$ $e(A)$ and $G(A)$ is reduced [3, Theorem 1]. But, By Lemma 2.1,(a) we have the inclusion $G(A) \subset G(A) \otimes_{k(\mathfrak{m})} K$. Hence $G(A) \otimes K$ is the extension to $K$ of the $k(\mathfrak{m})$ algebra $G(A)$ and is reduced if $k(\mathfrak{m})$ has characteristic zero. In general, if $A$ is seminormal, the closed point of $\operatorname{Spec}(A)$ needs not to be ordinary. In fact let $k$ be a field of characteristic two with algebraic closure $\bar{k}$. Let $b^{2}=a=$ $-a \in k$, with $b \in \bar{k}-k$. Set $A=\left(k[X, Y, Z] /\left(Y^{2}-a X^{2}-X^{3}\right)\right)_{(X, Y, Z)}$. Clearly $e(A)=\operatorname{emdim}(A)=2$ and $G(A)=k[X, Y] /\left(Y^{2}-a X^{2}\right)$ is reduced. Hence $A$ is seminormal. But $Y^{2}-a X^{2}=(Y+b X)^{2}$. Moreover $k(\mathfrak{m})=k$ and $K=\bar{k}$. Then $G(A) \otimes_{k(\mathfrak{m})} K=\bar{k}[X, Y] /(Y+b X)^{2}$ is not reduced.

Let $R=k\left[x_{0}, \ldots, x_{r}\right]$ be a reduced standard graded finitely generated $k$-algebra over an algebraically closed field $k$. Let $\operatorname{dim}(R)=1$ and set $e(R)=e$. Then $\operatorname{Proj}(R)=\left\{P_{1}, \ldots, P_{e}\right\} \subset \mathbb{P}^{r}$ is a finite set of points . Vice versa any set of projective points $\left\{P_{1}, \ldots, P_{e}\right\} \subset \mathbb{P}^{r}$ is equal to $\operatorname{Proj}(R)$ where $R$ is its homogeneous 
Definition 2.7. The set $\left\{P_{1}, \ldots, P_{e}\right\} \subset \mathbb{P}^{r}$ is in generic position in $\mathbb{P}^{r}$ (or the points $P_{1}, \ldots, P_{e}$ are in generic position in $\mathbb{P}^{r}$ ) if the Hilbert function of $R$ is maximal that is $H^{0}(R, n)=\operatorname{Min}\left\{e,\left(\begin{array}{c}n+r \\ r\end{array}\right)\right\}$ [11, Definition 3.1].

Remarks. 1. It is proved in [4, Theorem 4] that, for any $e$ and $r$, "generic position" is an open nonempty condition.

2. For details on the notion of points in generic position. see [11]

Example 2.8. It is easily seen that any set of points of $\mathbb{P}^{1}$ is in generic position.

Example 2.9. A set of $\left(\begin{array}{c}n+r \\ r\end{array}\right)$ points in $\mathbb{P}^{r}(n>0, r>0)$ is in generic position if and only if they do not lie on a hypersurface of degree $n$ [11, Corollary 3.4], in particular six points in $\mathbb{P}^{2}$ are in generic position if and only if they do not lie on a conic.

Let $S=R\left[T_{1}, \ldots, T_{n}\right], n \geq 0$, be the polynomial ring over a reduced standard graded finitely generated $k$-algebra $R=k\left[x_{0}, \ldots, x_{r}\right]$ ( $k$ algebraically closed). Let $\operatorname{dim}(R)=1$. Then $\operatorname{Spec}(S)=\left\{L_{1}, \ldots, L_{e}\right\} \subset \mathbb{A}^{n+r+1}$, is a set of linear varieties of dimension $n+1$ all containing a linear subvariety (through the origin) $L$ of dimension $n$. Vice versa any set of such linear varieties is equal to $\operatorname{Spec}(S)$ where $S$ is its affine coordinate ring.

Definition 2.10. The set of linear varieties $\left\{L_{1}, \ldots, L_{e}\right\}$ is in generic position in $\mathbb{A}^{n+r+1}$ if the set of points $\operatorname{Proj}(R)$ is in generic position in $\mathbb{P}^{r}$.

Example 2.11. A union of lines of $\mathbb{A}^{r+1}$ through the origin is in generic position if the corresponding set of projective points of $\mathbb{P}^{r}$ is in generic position. In particular, if $\operatorname{emdim}(A)=r+1$, the set of geometric tangents $\operatorname{Spec}\left(\left(G(A) \otimes_{k(\mathfrak{m})} K\right)_{\text {red }}\right) \subset \mathbb{A}^{r+1}$ of $\operatorname{Spec}(A)$ at its maximal ideal is in generic position in $\mathbb{A}^{r+1}$ if the Hilbert function of $\left(G(A) \otimes_{k(\mathfrak{m})} K\right)_{\text {red }}$ is maximal.

Theorem 2.12. Let emdim $(A)=r+1$. If the closed point of $\operatorname{Spec}(A)$ is ordinary with geometric tangents in generic position in $\mathbb{A}^{r+1}$ then the rings $G(B) \otimes_{k(\mathfrak{m})} K$ and $G(B)$ are reduced.

Proof. Set $D=G(A) \otimes K$ and $D_{\text {red }}=D / \operatorname{nil}(D)$. By the surjective graded homomorphism $\phi: D \rightarrow D_{\text {red }}$ we deduce that $H\left(D_{\text {red }}, n\right) \leq H(D, n)$, for any $n$. If we prove that $H\left(D_{\text {red }}, n\right)=H(D, n)$ then $\phi$ is an isomorphism and $D$ is reduced (and $G(A) \subset D$ [Lemma 2.1,(a)] is reduced). But $H\left(D_{\text {red }}, n\right) \leq H(D, n) \leq$ $\operatorname{Min}\left\{e,\left(\begin{array}{c}n+r \\ r\end{array}\right)\right\}$, and $H\left(D_{r e d}, n\right)=\operatorname{Min}\left\{e,\left(\begin{array}{c}n+r \\ r\end{array}\right)\right\}$, by assumption, whence the result.

Remark. If $A$ is the local ring at a point of a curve over an algebraically closed field $k$ then $k(\mathfrak{m})=k$ and $G(A) \otimes_{k(\mathfrak{m})} K=G(A)$. Hence, in this case Theorems 2.5 and 2.12 give the results of [10, Lemma-Definition 2.1 and Theorem 3.3]. Although, by Theorem 2.12, an ordinary point of a curve has, in general, reduced tangent cone, there are large classes of examples of ordinary points whose tangent cone is not reduced [9, Section 4].

\section{Ordinary CODIMENSION OnE SUbVarieties.}

In this section $X=\operatorname{Spec}(R)$ is a reduced variety over an algebraically closed field $k$ and $Y=\operatorname{Spec}(R / \mathfrak{q})$ is an irreducible codimension one subvariety $Y$ of $X$ 
field $k(\mathfrak{q})$ of $R$ at $\mathfrak{q}$. We say that $e\left(R_{\mathfrak{q}}\right)=e$ is the multiplicity of $Y$ (on $X$ ) and we set $\operatorname{emdim}\left(R_{\mathfrak{q}}\right)=r+1$. Note that, if $A$ is the local ring of $X$ at a closed point $x$ of $Y$ and $\mathfrak{p}=\mathfrak{q} A$ is the prime ideal in $A$ defining $Y$, then $A_{\mathfrak{p}}=R_{\mathfrak{q}}$. Hence $k(\mathfrak{q})$ is equal to the residue field $k(\mathfrak{p})$ of $A$ in $\mathfrak{p}$.

Definition 3.1. Let $A$ be the local ring of $X$ at a closed point $x$ of $Y$. Let $\gamma$ be a branch of $X$ at $x$ and $\mathfrak{p}$ the corresponding minimal prime of $A^{h}$ [Lemma 1.2]. $\operatorname{Spec}\left(G\left(A^{h} / \mathfrak{p}\right)\right)$ is the tangent cone to the branch $\gamma$ and $\operatorname{Spec}\left(G\left(A^{h} / \mathfrak{p}\right)_{\text {red }}\right)$ is the tangent cone, as a set, to $\gamma$. If the branch $\gamma$ is linear the ring $A^{h} / \mathfrak{p}$ is regular [Definition 1.3] and then its tangent cone coincides with the tangent space and we call it the tangent space to the branch $\gamma$.

Theorem 3.2. Let $x$ be a point of $Y$ and $A$ be the local ring of $X$ at $x$. Suppose $Y$ is nonsingular at $x$ and $X$ is normally flat along $Y$ at $x$. Let $\gamma$ be a branch of $X$ at $x$. Then $\operatorname{Spec}\left(G(A)_{\text {red }}\right.$ ) ( that is the tangent cone to $X$ at $x$, as a set) is union of linear varieties. Moreover the tangent cone to the branch $\gamma$ is, as a set, a linear variety of $\operatorname{Spec}\left(G(A)_{\text {red }}\right)$ and vice versa each linear variety of $\operatorname{Spec}\left(G(A)_{\text {red }}\right)$ is the tangent cone, as a set, of at least one branch.

Proof. Spec $\left(G(A)_{\text {red }}\right)$ is union of linear varieties if and only if the minimal primes of $G(A)_{\text {red }}$ are generated by linear forms. Moreover, if $\mathfrak{p}$ is the prime of $Y$ in $A$, $\left(G_{\mathfrak{p}}(A) \otimes_{A / \mathfrak{p}} k\right)_{\text {red }}$ is a one-dimensional reduced $k$-algebra over the algebraically closed field $k$ and then its minimal primes are generated by linear forms. But the isomorphism $G(A) \cong\left(G_{\mathfrak{p}}(A) \otimes_{A / \mathfrak{p}} k\right)\left[T_{1}, \ldots, T_{d}\right]$ of Theorem 1.6,(a) induces an isomorphism of graded $k$-algebras $G(A)_{\text {red }} \cong\left(G_{\mathfrak{p}}(A) \otimes_{A / \mathfrak{p}} k\right)_{\text {red }}\left[T_{1}, \ldots, T_{d}\right]$ and then also the minimal primes of $G(A)_{\text {red }}$ are generated by linear forms.

The second claim is proved in [2, Theorem 4.5].

Definition 3.3. Let $Y$ have multiplicity $e$ on $X$.

(a) A closed point $x$ of $Y$ is an ordinary point if, as a set, the tangent cone to $X$ at $x$ is the union of $e$ linear varieties;

(b) $Y$ is an ordinary subvariety of $X$ if the closed point of the one-dimensional scheme $\operatorname{Spec}\left(R_{\mathfrak{q}}\right)$ is ordinary.

Theorem 3.4. Let $Y$ have multiplicity $e$ on $X$ and $x$ be a closed point of $Y$. If $Y$ is nonsingular at $x$ and $X$ is normally flat along $Y$ at $x$, then $x$ is an ordinary point of $X$ if and only if $X$ has, at $x$, e linear branches with distinct tangent spaces. Moreover if these tangent spaces are in generic position then the tangent cone of $X$ at $x$ is reduced.

Proof. Let $A$ be the local ring of $X$ at $x$. If $x$ is an ordinary point of $X$ then $\operatorname{Spec}\left(G(A)_{\text {red }}\right.$ is the union of $e$ linear varieties [Definition 3.3] which by Theorem 3.2 are the tangent spaces of $e$ branches and these are linear by Proposition 1.4,(c). If $x$ has $e$ branches with distinct tangent spaces then, by Theorem 3.2 these are components of $G(A)_{\text {red }}$ and then $x$ is ordinary. Now we prove the second claim. Let $\mathfrak{p}$ be the prime of $Y$ in $A$ and consider the isomorphism $G(A)_{\text {red }} \cong\left(G_{\mathfrak{p}}(A) \otimes_{A / \mathfrak{p}}\right.$ $k)_{\text {red }}\left[T_{1}, \ldots, T_{d}\right]$ induced by the isomorphism of Theorem 1.6,(a). By Definition $2.10 \operatorname{Spec}\left(G(A)_{\text {red }}\right)$ is the union of $e$ linear varieties in generic position if and only if $\operatorname{Proj}\left(\left(G_{\mathfrak{p}}(A) \otimes_{A / \mathfrak{p}} k\right)_{\text {red }}\right)$ consists of points in generic position that is, if we set $D=G_{\mathfrak{p}}(A) \otimes_{A / \mathfrak{p}} k, H\left(D_{r e d}, n\right)=\operatorname{Min}\left\{e,\left(\begin{array}{c}n+r \\ r\end{array}\right)\right\}$ But the Hilbert function 
then $e=e(A)=e\left(G\left(A_{\mathfrak{p}}\right)\right)$. Hence $H(D, n) \leq \operatorname{Min}\left\{e,\left(\begin{array}{c}n+r \\ r\end{array}\right)\right\}$. Then $H\left(D_{\text {red }}, n\right)=$ $H(D, n)$, and $D=D_{\text {red }}$ is reduced. Thus also $G(A)$, which is isomorphic to a polynomial ring over $D$, is reduced.

Theorem 3.5. The following conditions are equivalent:

(a) $Y$ is an ordinary subvariety of $X$

(b) there exists an open nonempty subset $U$ of $Y$ such that every closed point $x$ of $U$ is ordinary.

(c) there exists an open nonempty subset $U$ of $Y$ such that $X$ has e linear branches with distinct tangent spaces, at any point of $U$.

Proof. Let $Y$ have multiplicity $e$ on $X$. Then $e\left(G\left(R_{\mathfrak{q}}\right) \otimes_{k(\mathfrak{q})} K\right)=e$ [Lemma 2.1,(c)].

$(a) \Rightarrow(b)$ If $Y$ is ordinary $\operatorname{Proj}\left(G\left(R_{\mathfrak{q}}\right) \otimes_{k(\mathfrak{q})} K\right)$ has $e$ points (that is $e$ irreducible components) [Definition 3.3,(b) and Theorem 2.5, $(a) \Rightarrow(b)]$. Then by $\left[6, N^{0} 28\right.$, Proposition 9.7.8] there exists an open nonempty subset $U_{1}$ of $Y$ such that, for every closed point $x$ of $U_{1}, G_{\mathfrak{p}}(A) \otimes_{A / \mathfrak{p}} k$ ( $A$ local ring of $X$ at $x$ ) has $e$ irreducible components that is $e$ points [see the proof of Theorem 2.5, $(b) \Leftrightarrow(a)$ ]. Then the $e$ minimal primes of $G_{\mathfrak{p}}(A) \otimes_{A / \mathfrak{p}} k$ are generated by linear forms. Furthermore, by Theorems 1.6 and 1.8, there exists an open nonempty subset $U_{2}$ of $Y$ such that, for every closed point $x$ of $U_{2}, G(A)$ is isomorphic to a polynomial ring over $G_{\mathfrak{p}}(A) \otimes_{A / \mathfrak{p}} k$. Then if $x$ is a point of $U_{1} \cap U_{2}$ the minimal primes of $G(A)$ are extensions of the minimal primes of the one dimensional finitely generated graded $k$-algebra $G_{\mathfrak{p}}(A) \otimes_{A / \mathfrak{p}} k$, hence they are generated by linear forms.

$(b) \Rightarrow(a)$ Suppose that, at every point $x$ of an open nonempty subset of $Y$, $\operatorname{Spec}\left(G(A)_{\text {red }}\right)$ has $e$ irreducible components, that is $G(A)_{\text {red }}$ has $e$ minimal primes. Then, by the graded isomorphism $G(A)_{r e d} \cong\left(G_{\mathfrak{p}}(A) \otimes_{A / \mathfrak{p}} k\right)_{r e d}\left[T_{1}, \ldots, T_{d}\right]$, on the points of an open set of $Y,\left(G_{\mathfrak{p}}(A) \otimes_{A / \mathfrak{p}} k\right)_{\text {red }}$ has $e$ minimal primes $\left[6, N^{0} 28\right.$, Proposition 9.7.8]. Hence the zero-dimensional scheme $\operatorname{Proj}\left(G\left(A_{\mathfrak{p}}\right) \otimes_{k(\mathfrak{p})} K\right)=$ $\operatorname{Proj}\left(G\left(R_{\mathfrak{q}}\right) \otimes_{k(\mathfrak{q})} K\right)$ has $e$ points, and then, since by Lemma 2.1,(c) $e\left(G\left(A_{\mathfrak{p}}\right) \otimes_{k(\mathfrak{p})}\right.$ $K)=e\left(G\left(A_{\mathfrak{p}}\right)\right)=e\left(A_{\mathfrak{p}}\right)=e$, is reduced [Theorem 2.5, $(b) \Rightarrow(a)$ ].

$(b) \Leftrightarrow(c)$ It is an easy consequence of Theorem 1.8 and Theorem 3.4.

Let $Y$ be an ordinary subvariety of codimension one and of multiplicity $e$ on a reduced variety $X$. Let $x$ be a closed point of $Y$ such that $Y$ is nonsingular at $x$ and $X$ is normally flat along $Y$ at $x$. Then by Theorem 3.2 the tangent cone of $X$ at $x$ consists, as a set, of linear varieties, but the number of these can be less then $e$ and then $x$ is not an ordinary point of $X$ as the following example shows.

Example 3.6. Let $R=\mathbb{C}\left[X_{1}, X_{2}, X_{3}\right] /\left(X_{1} X_{2}^{n}-X_{3}^{n}\right)=\mathbb{C}\left[x_{1}, x_{2}, x_{3}\right](n \geq 2)$ and $A$ be the local ring of $X=\operatorname{Spec}(R)$ at the maximal ideal $\left(x_{1}, x_{2}, x_{3}\right)$. The nonnormal locus of the hypersurface $X$ is the line $Y: x_{2}=0, x_{3}=0$ of multiplicity $n$ on $X$ and if $a \in \mathbb{C}, a \neq 0$, the tangent cone at the point $(a, 0,0)$ of $L$ consists, as a set, of the $n$ distinct planes $x_{3}=b x_{2}$, where $b^{n}=a$. Then $Y$ is an ordinary subvariety of $X$. Moreover $e(A)=e\left(R_{\mathfrak{q}}\right)=n$, where $\mathfrak{q}=\left(x_{2}, x_{3}\right)$. Then, by [7, Corollary (23.22)], $X$ is normally flat along $Y$ at $x$ and clearly $Y$ is non singular at $x$. But the tangent cone of $X$ at $(0,0,0)$ is $\operatorname{Spec}\left(\mathbb{C}\left[X_{1}, X_{2}, X_{3}\right] /\left(X_{3}^{n}\right)\right.$ and then, as a set, consists of the plane $x_{3}=0$. But $e=e(A)=n \geq 2$ and $(0,0,0)$ is not 


\section{REFERENCES}

[1] C. Cumino, On the order of branches, in "Commutative Algebra" (Marcel Dekker, ed.), Lecture Notes in Pure and Applied Mathematics, vol. 84, 1981, pp. 49-64.

[2] C. Cumino, Tangent cones and analytic branches, Rev. Roumaine Math. Pures Appl. 31 (1986), 843-854.

[3] E.D Davis, On the geometric interpretation of seminormality, Proc. Am. Math. Soc. 68 (1978), 1-5.

[4] A.V. Geramita, F. Orecchia, On the Cohen-Macaulay type of s-lines in $\mathbb{A}^{n+1}$, J. Algebra 70 (1981), 116-140.

[5] S. Greco, On the theory of branches, Int. Symp. of Algebraic Geometry, Kioto (1977), 477-493.

[6] A. Grothendieck, J. Dieudonné, Éléments de Géométrie Algébrique, Publ. Math. I.H.E.S. Ch IV (1964-1967).

[7] M. Herrmann, S. Ikeda, U. Orbanz, Equimultiplicity and blowing up, Springer-Verlag, 1988.

[8] H. Hironaka, Resolution of singularities of an algebraic variety over a filed of characteristic zero, Ann. Math. 79 (1964), 109-326.

[9] F. Orecchia, One-dimensional local rings with reduced associated graded ring and their Hilbert function, Manuscripta Math. 32 (1980), 391-405.

[10] F. Orecchia, Ordinary singularities of algebraic curves, Can. Math. Bull. 24 (1981), 423-431.

[11] F. Orecchia, Points in generic position and conductors of curves with ordinary singularities, J. London Math. Soc. 24 (1981), 85-96.

[12] F. Orecchia, Points in generic position and conductor of varieties with ordinary multiple subvarieties of codimension one, J. of Pure and Appl. Algebra, to appear.

[13] M. Raynaud, Anneaux locaux henséliens, Lecture Notes Math. 169 (1969), Springer Verlag.

Ferruccio Orecchia, Dipartimento di Matematica e Applicazioni "R. Caccioppoli", Complesso Universitario di Monte S. Angelo - Via Cintia , 80126 Napoli-Italy

E-mail address: ORECCHIA@MATNA2.DMA. UNINA.IT . 